\title{
LE MAL RADICAL CHEZ FICHTE ENTRE KANT ET SCHELLING
}

Claude Piché, Université de Montréal

Ceci est la version «postpublication » d'un article paru dans Symposium, vol. 3, no. 2, 1999, p. 209-231.

RÉSUMÉ : Dans cet article, je soumets à une discussion critique deux thèses à propos du mal radical chez Fichte : 1- la thèse selon laquelle sa conception ne serait pas foncièrement différente de celle de Kant (position défendue par A. Soller et M. Ivaldo) et 2- la thèse selon laquelle c'est seulement après l'arrivée au pouvoir de l'empereur Napoléon, incarnant l'auto-affirmation absolue du libre arbitre, que la position de Fichte se serait rapprochée de la conception kantienne (R. Lauth). Or je marque mes distances face à ces deux thèses en insistant sur le fait que, tout au long de sa carrière, Fichte a défendu une conception du mal radical, qu'il appelle d'ailleurs de manière très révélatrice « radikales Übel », fondée sur l'inertie et l'ignorance.

MOTS-CLÉS : Fichte, Kant, Napoléon, auto-affirmation, Böse/Übel, ignorance, paresse

\begin{abstract}
In this article, I submit to critical analysis two theses concerning radical evil in Fichte: 1- the thesis according to which his conception would not fundamentally differ from Kant's position (a thesis defended by A. Soller and M. Ivaldo) and 2- the thesis according to which it is only after the seizure of power by the emperor Napoléon, representing the absolute self-assertion of the free will, that Fichte's position would have come closer to Kant's (R. Lauth). I would like to distance myself from both theses by stressing the fact that all through his career Fichte has been the proponent of a conception of radical evil, that he revealingly calls "radiakles Übel," based on inertia and ignorance.
\end{abstract}

KEYWORDS : Fichte, Kant, Napoléon, self-assertion, Böse/Übel, ignorance, laziness

Dans un passage crucial de son traité de 1809 sur l'Essence de la liberté humaine, Schelling n'hésite pas à rejeter le parti de Fichte pour adopter celui de Kant, et ce sur la question centrale du traité: la liberté. En effet, Schelling reconnaît à Kant le mérite d'avoir décelé que le mal moral dépend directement d'un acte de liberté, point de vue auquel Fichte, pourtant considéré comme le chantre de la liberté, a été incapable de s'élever. Voici le passage en question:

Seul ce mal résultant d'un acte propre, mais contracté dès la naissance, peut être nommé le mal radical, et il est remarquable que Kant, qui ne s'était point élevé dans sa philosophie théorique jusqu'à l'idée d'un acte transcendantal déterminant l'être de l'homme en son tout, ait été conduit dans ses recherches ultérieures, par le seul fidèle examen des phénomènes du jugement moral, à reconnaître aux actions humaines un fondement, comme il dit, subjectif, qui précède tout acte tombant sous les sens et doit 
pourtant nécessairement être lui aussi un acte de liberté, alors que Fichte, qui avait conçu en philosophie spéculative l'idée d'un tel acte, retomba avec la Sittenlehre dans le philanthropisme dominant, et ne voulut trouver ce mal antérieur à tout agir empirique que dans l'inertie de la nature humaine ${ }^{1}$.

La question qui se pose d'emblée consiste à savoir si l'accusation de philanthropisme lancée à Fichte n'est que circonstancielle, ou si elle touche la chose même. On sait qu'à partir de 1800 les dissensions qui troublaient jusque-là les relations personnelles entre Fichte et Schelling s'expriment au grand jour, et on pourrait croire que la flèche décochée dans l'extrait ci-dessus n'est qu'un élément de plus de ce débat devenu acerbe.

Pourtant il n'en est rien, car le problème soulevé par Schelling est trop important pour qu'il puisse être ramené à un conflit de personnalités. La liberté, non dans son sens formel mais dans sa signification réelle, renvoie pour Schelling à la capacité de faire le bien et le mal. Or, le jugement qu'il porte sur les théories du mal radical de Kant et de Fichte est intimement relié à son propre projet et il y a lieu d'accorder à la différence qu'il fait entre les deux penseurs toute l'attention voulue.

La préférence sans équivoque manifestée à l'endroit de Kant appelle pourtant dès le départ quelques précautions. Ainsi dans ce qui constitue le locus classicus du thème du mal radical chez Fichte, le Système de l'éthique de 1798, celui-ci prétend que la théorie qu'il nous livre n'est, tout bien considéré, que le fin mot de la conception kantienne. Dès lors, de deux choses l'une: ou bien Fichte a raison et l'accusation de philanthropisme de Schelling serait bel et bien à mettre au compte de la polémique; ou bien c'est Schelling qui a vu juste et Fichte se méprend sur ses affinités avec la position kantienne --à moins qu'il ne veuille masquer le différend qui l'oppose à Kant.

Dans un premier temps, nous allons examiner la position d'Alois Soller et de Marco Ivaldo, qui estiment tous deux que Fichte dit vrai et que ses idées sur le mal radical s'inscrivent effectivement dans la droite ligne de celles de Kant. Dans un second temps, nous nous interrogerons sur la constance de la théorie fichtéenne du mal radical à travers toute son oeuvre. Car il se pourrait fort bien qu'au moment où, en 1809, Schelling formule sa critique à l'endroit de la Sittenlehre de 1798, l'argument ne tienne plus. En d'autres mots, il se pourrait que, malgré certaines divergences initiales, Fichte en soit venu finalement à se rallier à Kant sur la question de l'imputabilité du mal et sur le rôle qu'y joue la liberté. C'est du moins la question qu'il est

\footnotetext{
${ }^{1}$ F. W. J. Schelling, Philosophische Untersuchungen über das Wesen der menschlichen Freiheit und die damit zusammenhängenden Gegenstände (1809), édition Cotta, Sec. I/7, p. 388-389; tr. (modifiée) J.-F. Courtine et E. Martineau, Recherches philosophiques sur l'essence de la liberté humaine et les sujets qui s'y rattachent, dans Schelling, Oeuvres métaphysiques (1805-1821), Paris, Gallimard, 1980, p. 172 (nos italiques).
} 
permis de soulever s'il faut en croire la thèse soutenue par Reinhard Lauth dans sa conférence intitulée « L'action historique d'après la philosophie transcendantale de Fichte ». Cela signifierait, selon cette thèse formulée, il est vrai, sans référence à l'accusation de Schelling, que les trois penseurs s'entendraient à la fin sur le rôle dévolu à la liberté dans la genèse du mal moral. Ce qui est loin d'être le cas, comme nous allons le voir.

\section{I - La conception du mal moral chez Fichte est-elle kantienne?}

Sur un point essentiel, il est évident que Fichte est entièrement d'accord avec Kant. Tous deux renoncent en effet à chercher une explication métaphysique au mal moral en tentant, par exemple, de l'attribuer aux limites intrinsèques de la créature. Pour eux, le mal radical relève strictement de la liberté. C'est dire que pour les deux auteurs, le mal doit être imputable à l'homme, et à lui seul. En cela Soller et Ivaldo décèlent, à juste titre, une communauté de vues entre Fichte et Kant et ont tout à fait raison de souligner l'inspiration kantienne de cette phrase de Fichte: «...s'il se trouve une maxime mauvaise [böse], on ne doit pas l'expliquer autrement que par la liberté de l'homme lui-même et celui-ci ne peut en reporter la faute [Schuld] sur quelque chose d'extérieur à lui ${ }^{2}$ ». La ligne de conduite est donc à cet égard la même pour Kant et Fichte, mais la question demeure: les deux théories du mal radical coïncident-elles parfaitement? Il faut en convenir à la suite d'Ivaldo, les textes de Fichte, tout autant d'ailleurs que ceux de Kant, ne sont pas dépourvus d'ambiguïtés. Ivaldo choisit pour sa part d'exposer les points de convergence. Pour ma part, j'adopterai le parti opposé, et pas simplement pour faire bonne mesure, mais pour montrer que le différend entre Fichte et Kant est plus important, pour ne pas dire plus radical, qu'il n'y paraît.

En fait, la question du mal radical ne constitue pas un point de doctrine secondaire à l'intérieur de la théorie morale. Elle engage en vérité la conception de la liberté dans ce qu'elle a de plus spécifique chez les deux auteurs. Il ne faut donc pas s'étonner de voir Fichte, au $§ 16$ de sa Sittenlehre de 1798, se référer à deux reprises à sa « doctrine de la liberté ${ }^{3} »$. Pour le formuler dans les termes de la phrase précitée, il y va du rapport qu'entretient la « mauvaise maxime »

\footnotetext{
${ }^{2}$ J. G. Fichte, Collegium über die Moral im Sommerhalben Jah. 1796, GA IV/1, p. 89. Voir la reprise presque textuelle dans le System der Sittenlehre nach den Principien der Wissenschaftslehre (1798), SW IV, p. 180; tr. P. Naulin, Le système de l'éthique d'après les principes de la Doctrine de la Science, Paris, PUF, 1986, p. 173.

Alois K. Soller, Trieb und Reflexion in Fichtes Jenaer Philosophie, Würzburg, Königshausen \& Neumann, 1984, p. 145. Marco Ivaldo, « Das Problem des Bösen bei Fichte », Fichte-Studien, 3 (1991), 159-160.

${ }^{3}$ Fichte, System der Sittenlehre (1798), p. 181, 198; tr. p. 175, 190.
} 
avec la « liberté ». Évidemment, et nous allons avoir le loisir d'y insister à nouveau, le choix de la mauvaise maxime doit être en tant que tel imputable. Mais nous allons voir que, contrairement à ce que l'on retrouve chez Kant, la prévalence de la mauvaise maxime est attribuable chez Fichte à une absence de liberté. C'est là que se creuse le fossé entre les deux penseurs. Dans ce qui suit, nous allons procéder à l'analyse de la position de Fichte à la lumière du $\$ 16$ du Système de l'éthique en nous référant aussi à la Destination de l'homme, texte qui vient clore, pour ainsi dire, la période d'Iéna. Nous procéderons en trois étapes, chacune d'elles gravitant autour d'une thèse relative au thème de la liberté dans son rapport à la question du mal radical. Ainsi, premièrement, la théorie fichtéenne de la liberté offre un avantage théorique marqué sur celle de Kant en ce qu'elle se présente comme une énergétique. Deuxièmement, la philosophie de Fichte a beau reposer tout entière sur la primauté de la raison pratique, la conception du mal moral qui s'en dégage n'en comporte pas moins une dimension cognitive qui l'apparente aux théories du rationalisme classique. Enfin, troisièmement, j'entends soutenir la thèse selon laquelle la théorie de la liberté qui se trouve au fondement de la Doctrine de la Science se règle sur un modèle politique, ce qui permet d'associer Fichte au mouvement de l'Aufklärung plus étroitement encore qu'il n'est possible de le faire avec Kant.

Si la question du mal radical est directement tributaire de la théorie de la liberté, il faut comprendre qu'aux yeux de Fichte la version kantienne de cette théorie pose de sérieux problèmes. À vrai dire, c'est là que s'installe d'emblée le différend, avec les conséquences que nous allons voir. Pour le dire brièvement, Fichte n'a jamais pu se laisser convaincre par la solution du problème de la liberté transcendantale exposée dans la première Critique. On se souvient que la solution critique apportée au troisième conflit de l'Antinomie de la raison pure mise de façon très explicite sur un dualisme. Il y est question de deux types de causalité, l'une libre, l'autre nécessaire, occupant chacune un registre séparé: l'une dans le monde intelligible, l'autre dans le monde sensible. Ce qui conduit Kant à postuler en l'homme un caractère intelligible et un caractère empirique. Or, Fichte a de la difficulté à accepter une théorie qui pour un seul et même phénomène dans le monde sensible fait intervenir deux principes d'explication distincts (liberté et nécessité naturelle), pouvant entrer en concurrence sans toutefois que l'un interfère avec l'autre. En d'autres mots, Fichte ne voit pas comment à l'intérieur du kantisme on peut parvenir à comprendre de quelle manière une causalité libre intervient dans le monde empirique. Il est à ce point convaincu de l'échec de Kant dans la Critique de la raison pure qu'il pense que celui-ci est encore aux prises avec le même problème dans la troisième Critique, notamment dans l'Introduction ${ }^{4}$. Kant, pourtant, semble satisfait de sa solution critique de la

\footnotetext{
${ }^{4}$ Fichte a toujours considéré que l'intervention de la liberté nouménale sur le terrain de l'expérience empirique ne pouvait être pour Kant qu'une thèse « provisoire». Voici un exemple des difficultés rencontrées par Fichte dans la théorie kantienne de la liberté: "Pour l'être-déterminé comme phénomène,
} 
troisième Antinomie, au point de s'appuyer sur celle-ci tant dans la seconde Critique que dans l'essai de 1792 intitulé « De l'immanence du mauvais principe au bon ou sur le mal radical dans la nature humaine », essai qui, comme on sait, deviendra la première partie de l'ouvrage sur la Religion. Dès lors, si Kant, aux yeux de Fichte, n'a pas encore réussi en 1792 à surmonter les apories de son dualisme, il va de soi qu'une nouvelle approche du problème de la liberté s'impose, ce qui donne le coup d'envoi de la Doctrine de la Science.

Il ne faut pas oublier que le $\S 16$ de la Sittenlehre peut légitimement faire usage des résultats de la Doctrine de la Science, comme le titre même de l'oeuvre l'y autorise: Système de l'éthique selon les principes de la Doctrine de la Science. C'est dire que les acquis de cette nouvelle philosophie transcendantale centrée explicitement sur la spontanéité du Moi doivent être mis à profit pour l'éthique, au risque d'envisager à nouveaux frais le problème du mal moral. En effet, lorsqu'il s'agit de surmonter la dichotomie kantienne entre intelligible et sensible, la Doctrine de la Science offre l'avantage de mettre sur le même plan la liberté et la nature, ou encore le Moi et le Non-Moi. La liberté et la nature n'étant plus désormais hétérogènes, comme chez Kant, elles peuvent entrer en contact: la concurrence entre ces deux instances peut à présent s'exercer sur le même terrain, celui du Moi, le Non-Moi n'étant pas foncièrement extérieur au Moi. Telle est l'avancée théorique de la Doctrine de la Science par rapport au kantisme ${ }^{5}$. Bien sûr, si le Non-Moi incarne désormais la nature, la nécessité propre à celui-ci ne s'exerce pas à la

il faut supposer d'après la loi de la causalité naturelle un fondement réel effectif [wirklicher Realgrund] dans un phénomène antérieur. Or, que l'être-déterminé par la causalité de la nature et la détermination par la liberté cö̈ncident - ce qui doit tout aussi bien être admis au profit d'un ordre moral du monde - ceci n'a de fondement ni dans la nature, qui n'a aucune causalité sur la liberté, ni dans la liberté, qui n'a aucune causalité dans la nature. Il faut plutôt rechercher cette coïncidence dans une loi supérieure qui contient les deux en elle et les unit, à la manière d'une harmonie préétablie des déterminations par la liberté avec les déterminations par la loi naturelle. " Recension von Creuzers skeptischen Betrachtungen über die Freiheit des Willens (1793), SW VIII, p. 414-415.

Frederick Neuhouser a bien su déceler l'équivoque sur laquelle repose le lecture fichtéenne de la troisième Critique de Kant:« ...le problème de Fichte devient celui de comprendre comment un sousensemble d'actions - les actions morales comme événements dans le monde empirique - peut à la fois faire l'objet d'une détermination causale et être considéré comme la conséquence d'une libre détermination de la volonté. En dépit de l'importance de cet enjeu, le fait est qu'il s'agit d'un problème différent de celui que Kant cherchait à solutionner dans la troisième Critique. » Fichte's Theory of Subjectivity, Cambridge, Cambridge University Press, 1990, p. 18.

${ }^{5}$ Voir à ce sujet, Peter Rohs, Johann Gottlieb Fichte, Munich, C. H. Beck, 1991, p. 104. Pour sa part, Teodor Oiserman estime qu'à son tour la philosophie de Fichte se montre incapable de comprendre le principe de la liberté lorsque celui-ci est incarné dans un sujet « empirique ». Ce en quoi précisément l'approche schellingienne se montre supérieure à la sienne. «Zur Frage der Differenz der Freiheitsphilosophie Schellings und der Freiheitslehren Kants und Fichtes », dans Schellings Weg zur Freiheitsschrift. Legende und Wirklichkeit. H. M. Baumgartner et W. Jacobs (dir.), Stuttgart/Bad Cannstatt, Frommann-Holzboog, 1996, p. 306-307. 
façon d'une contrainte mécanique sur le Moi: jamais un être libre ne saurait être soumis à un tel déterminisme. Il n'y a ici d'autre nécessité que morale. Or, ce résultat obtenu au plan de la spéculation offre non seulement une solution aux difficultés inhérentes au dualisme, voire au parallélisme kantien, il fournit également une explication de la liberté morale qui rend intelligible la présence du mal dans le monde. Alors qu'à l'égard du bien et du mal le sujet kantien opère par décrets insondables, la liberté du sujet moral fichtéen s'insère dans un rapport dynamique qui assigne à la nature (Non-Moi) un rôle de plein droit.

La définition du mal radical développée au $\$ 16$ de la Sittenlehre est bien connue: le mal moral réside dans l'inertie. Cette définition ne prend toutefois son sens que lorsqu'on l'insère dans la structure Moi/Non-Moi. Si le Moi est pure spontanéité, l'inertie en revanche ne peut se retrouver que du côté du Non-Moi, lequel limite le Moi dans son action en lui offrant une résistance. C'est pourquoi Fichte souligne que l'inertie n'est pas absence d'énergie. Au contraire, dans la mesure où le Non-Moi neutralise l'action d'un être qui est essentiellement spontanéité, elle apparaît comme une force (Kraft), un contrepoids, que le Moi doit s'employer à surmonter. On le voit, la liberté est ici conçue dans les termes d'une énergétique: le Moi doit puiser en lui-même la force de s'affirmer comme être libre. Certes, la liberté constitue l'essence du Moi pur qui est spontanéité, auto-position; mais en revanche le Moi empirique, objet de l'éthique, ne peut s'y élever que graduellement et par ses propres forces en repoussant en lui le Non-Moi. On retrouve ici dans la sphère de l'éthique le rapport quantitatif entre Moi et Non-Moi exposé en 1794 dans le troisième principe de la Doctrine de la Science. Aussi, en 1798, la liberté n'apparaît-elle pas comme un acquis, mais bien comme l'objet d'une conquête: le Moi empirique doit tendre à coïncider avec le Moi pur.

Dans ces conditions, il ne faut pas s'étonner de voir qu'au début du paragraphe qui nous occupe, Fichte envisage la question du mal moral du point de vue de l'histoire de l'humanité. L'homme comme sujet empirique est un être fini pour qui l'atteinte de la liberté morale se présente comme une tâche qui se réalise dans le temps. Si la liberté constitue l'essence de l'homme, encore faut-il qu'il la réalise en lui, qu'il s'y élève graduellement: telle est la nature de la tâche morale. À ce chapitre, Fichte nous livre les lignes de force de ce développement moral qui doivent guider notre lecture du cours de l'histoire. À une phase qui correspond au stade « animal », où l'instinct naturel dicte aveuglément la conduite, succède une phase qu'il caractérise comme celle de l' « animal intelligent », capable de choisir entre divers objets de satisfaction. Enfin, faisant suite à une phase intermédiaire d'affirmation égoïste de soi, le sujet accède à la liberté proprement dite en produisant en lui-même la loi morale. Ainsi, du point de vue de l'histoire de l'homme comme être fini, la liberté effective coïncide avec l'accession à la loi morale et à sa reconnaissance, alors que le stade initial, où ne prévaut que l'instinct naturel, 
représente le degré zéro de la liberté, c'est-à-dire le stade où l'inertie (Non-Moi) règne en maître. Et, puisque la définition du mal radical se ramène pour Fichte à l'inertie, il faut en conclure que le mal radical correspond pour l'homme à un état concret de non liberté. Il devient dès lors manifeste que l'on s'éloigne de la conception kantienne selon laquelle le mal radical est choisi librement, comme l'enseigne du moins l'expérience. J'ai souligné en commençant que la différence entre Fichte et Kant tient à la manière dont le mal se rapporte à la liberté; or on voit ici que pour Fichte le mal n'est pas l'objet d'une libre décision, mais plutôt la conséquence d'une absence de liberté.

Contrairement à ce que font Soller et Ivaldo, il y a lieu de cerner très précisément ce point de désaccord, sous peine de laisser échapper la spécificité et l'originalité de l'explication fichtéenne. Cette spécificité n'est pas toujours aisément repérable dans la mesure où Fichte laisse planer une certaine ambiguïté dans ses textes. Ainsi, par exemple, Ivaldo relève-t-il dans la Destination de l'homme cette expression, qui plaide en faveur d'une lecture kantienne du problème du mal: celui-ci serait imputable à un « mauvais usage de la liberté » (Missbrauch der Freiheit). On y retrouve également la référence à un « usage de la liberté pour le mal » (Gebrauch der Freiheit zum Bösen). Cependant, à la fin de son parcours dans la troisième section de l'ouvrage, Fichte expose clairement en quoi consiste un tel usage de la liberté: à tout prendre, il s'agit d'une renonciation à se prévaloir de sa liberté. Et Fichte de conclure: le mal est attribuable à un « manque de liberté » (Mangel an Freiheit) dû au fait que l'on cède sous le poids de l'inertie ${ }^{6}$. L'imputabilité tient strictement à ceci que le sujet moral a renoncé à fournir l'effort de s'élever à la loi morale. Il en allait de même, au demeurant, au §16 de la Sittenlehre qui, donnant parfois l'impression que le mal est l'objet d'un choix libre, stipule en toutes lettres que le mal résulte d'un « non usage » (Nichtgebrauch ${ }^{7}$ ) de la liberté. Il devient manifeste par là que le mal est imputable à une absence, à un manque. Il ne s'agit pas toutefois ici d'un retour à une conception métaphysique du mal qui chercherait une explication dans des carences inhérentes à l'état initial de la création. L'explication est de part en part morale, tout en prenant appui sur l'idéalisme transcendantal fichtéen: le Moi est en butte à une force d'inertie qui constitue une entrave à sa spontanéité. Mais il ne tient qu'à lui de contrer cette force en se déterminant à l'action. Nous parvenons de la sorte au résultat suivant: si la théorie kantienne du mal radical attribue celui-ci à un choix délibéré mais inexplicable, la théorie fichtéenne, bien qu'elle soit plus cohérente dans la manière dont elle met en scène les forces en présence, reporte le mystère à un

\footnotetext{
${ }^{6}$ Fichte, Die Bestimmung des Menschen (1800), SW II, p. 307, 276, 314. Cf. Marco Ivaldo, »Das Problem des Bösen bei Fichte«, p. 160.

${ }^{7}$ Fichte, System der Sittenlehre (1798), SW IV, p. 182; tr., p. 176.
} 
autre niveau: comment comprendre en effet qu'un être libre fini puisse se résoudre à passer du stade de l'inertie naturelle à celui de la liberté?

Pour prendre la mesure de la difficulté qui se présente ici, il faut passer à notre seconde thèse, qui a trait à la dimension cognitive, voire intellectualiste, de la conception fichtéenne de la moralité. Il va de soi qu'il serait vain de contester la primauté de la philosophie pratique chez Fichte, pas plus qu'il y a lieu de contester la dimension somme toute volontariste de sa morale. C'est d'un geste délibéré que le Moi secoue son inertie pour accéder à la liberté. Cependant, l'inertie ne touche pas que l'incapacité d'agir, elle concerne aussi l'« inertie originaire à l'égard de la réflexion ${ }^{8} »$. Il s'agit de la paresse qui empêche l'agent moral de s'élever à la pleine conscience de sa destination. En ce sens, Miklos Vetö parle fort judicieusement d' « inertie intellectuelle ${ }^{9} »$. C'est que l'être raisonnable fini en proie à la torpeur ne s'élève pas à la conscience de la loi. Voilà l'origine première du mal. La non-liberté dont il vient d'être question implique en tout premier lieu un état d'inconscience. Assumer sa liberté signifie d'abord et avant tout que 1 'on accède à la conscience de la $10 i^{10}$.

Une telle conception soulève au moins trois questions:

1- L'ignorance de la loi morale par le sujet contribue-t-elle à le disculper?

2- La conscience claire de la loi morale, qui coïncide avec le stade de la liberté pleinement assumée, comporte-t-elle la possibilité de contrevenir à la loi?

3- (en guise de corolaire:) Qu'en est-il de la possibilité de la perversion morale chez Fichte?

À la question de savoir si l'inconscience en regard de la loi morale contribue à innocenter le sujet en proie à l'inertie, Fichte répond par la négative. Le sujet qui ne s'élève pas à la conscience de la loi morale, laquelle seule lui permet pourtant de distinguer le bien du mal, est responsable de sa propre indolence. En clair, l'homme qui n'est pas libre est responsable de son propre esclavage. Ce qui signifie en vérité: l'homme qui ne s'élève pas à la conscience de la loi morale est coupable de cette ignorance elle-même. La faute est sienne, même si à ce stade il ne peut en être « conscient ${ }^{11} »$.

\footnotetext{
${ }^{8}$ Fichte, System der Sittenlehre (1798), SW IV, p.199; tr., p. 191.

${ }^{9}$ Miklos Vetö, De Kant à Schelling. Les deux voies de l'Idéalisme allemand. Grenoble, Millon, 1998, p. 397.

${ }^{10}$ Fichte, System der Sittenlehre (1798), SW IV, p. 199; tr., p. 191: « Nous supposons donc une inertie originaire à l'égard de la réflexion et, par voie de conséquence, à l'égard de l'action qui se règle sur cette réflexion. Ce serait donc là un mal radical [radikales Übel], véritable et positif, non quelque chose qui soit simplement négatif, comme il a semblé jusqu'à présent. »

${ }^{11}$ Fichte, System der Sittenlehre (1798), SW IV, p. 182; tr., p. 176: « En ce sens, le mal est inné à l'homme. Cependant, il n'est pas nécessaire qu'il en reste là, puisqu'il n'y a rien non plus qui le retienne
} 
La seconde question porte sur la contrepartie positive de la réponse précédente: est-il possible en toute conscience de passer outre à l'injonction de l'impératif moral? À nouveau, la réponse de Fichte est sans équivoque.

Il est absolument impossible et contradictoire que quelqu'un ayant une claire Conscience de son devoir à l'instant d'agir, se décide, avec bonne conscience, à ne pas faire son devoir...

La chose se passe donc ainsi: si l'on réfléchit continuellement à l'exigence de la loi, si celle-ci demeure devant les yeux, il est impossible de ne pas agir selon cette exigence et de la contredire. Si elle $s$ 'obscurcit, il est impossible d'agir en nous réglant sur elle ${ }^{12}$.

On mesure aisément la distance qui sépare ces affirmations de la théorie kantienne du mal radical. Pour Kant, en effet, la condition sine qua non pour commettre le mal moral, c'est la conscience claire du devoir, en sorte que la dérogation à la loi a lieu en toute connaissance de cause comme en témoigne cette définition standard tirée de l'essai sur le mal radical:

La proposition: 1'homme est mauvais, ne peut, d'après ce qui précède, avoir un autre sens que celui-ci: il est conscient [bewusst] de la loi morale et il a cependant admis dans ses maximes une occasionnelle déviance par rapport à elle ${ }^{13}$.

Si dans son texte Kant distingue trois degrés dans la progression vers le mal radical (fragilité, impureté et corruption), il ne manque pas de souligner à chacune de ces étapes que la pleine conscience du devoir est requise. Et c'est en ce sens qu'à ses yeux le choix du mal relève de la liberté. En d'autres termes, il n'y a pas d'imputabilité si le choix n'est pas éclairé.

La situation est, on s'en rend compte, toute différente chez Fichte. Encore que le mal moral corresponde à une absence de liberté, c'est-à-dire à l'absence de la conscience de la loi $\left(\right.$ Gesetzlosigkeit $\left.^{14}\right)$, il n'en reste pas moins imputable. Par ailleurs, dès lors que la liberté atteint sa pleine effectivité, au moment donc où la loi du devoir est clairement présente à la conscience, le choix du mal n'est plus possible.

en ce point. Il lui est tout aussi possible de se placer aussitôt au point de vue le plus élevé et, s'il ne l'a pas fait, c'est faute d'avoir employé [Nichtgebrauch] sa liberté, bien que, dans son état présent, il ne prenne pas conscience de sa culpabilité. Dans cette mesure, le mal en l'homme se fonde sur la liberté. »

${ }^{12}$ Fichte, System der Sittenlehre (1798), SW IV, p. 191, 192; tr. (modifiée), p. 184 (nos italiques).

${ }^{13}$ Immanuel Kant, Die Religion innerhalb der Grenzen der blossen Vernunft, AK VI, p. 32; tr. A. Philonenko, La religion dans les limites de la simple raison, dans Kant, Oeuvres philosophiques III, Paris, Gallimard, Biblioth. de la Pléiade, 1986, p. 45.

${ }^{14}$ Fichte, System der Sittenlehre (1798), SW IV, p. 183; tr., p. 176. 
Ce qui nous conduit à notre troisième question, relative à la possibilité d'une corruption de la nature humaine. On le sait, cette possibilité est admise par Kant. Mais, étant donné ce que nous venons de dire de l'impossibilité du choix conscient du mal chez Fichte, il est à prévoir que la perversion de la conviction morale ne trouvera guère de place dans sa doctrine. Et ceci s'annonce déjà dans les choix terminologiques opérés par Fichte: si l'expression « radikales Böse $^{15} \gg$ intervient à deux reprises au $\S 16$, c'est à chaque fois pour désigner la conception kantienne. Pour sa part, Fichte préfère avoir recours à la formule « radikales Übel ${ }^{16} »$. La nuance peut sembler superfétatoire, mais elle prend tout son sens quand on se souvient que Kant dans la Critique de la raison pratique avait pris soin de souligner la supériorité de la langue allemande qui permet de distinguer dans le malum latin les deux concepts: Böse et Übel ${ }^{17}$ (méchanceté/malheur). Et cela signifie que Fichte n'entend accorder dans son éthique aucune place à la méchanceté, attitude qui consisterait à prendre le contrepied de la loi.

Évidemment, la position de Fichte ne diffère en rien de celle de Kant lorsqu'il s'agit de refuser à l'homme l'accès au diabolique: « Aucun homme n'aime le mal [das Böse] parce que c'est le mal $^{18} »$. Cependant, la perversion (Verkehrtheit) que Kant est prêt à reconnaître à l'homme est d'un autre ordre. En fait, elle est plus banale puisqu'elle consiste seulement, comme il ressort de l'extrait de l'essai sur le mal précité, à s'accorder la possibilité de faire une exception à l'observance de la loi, et ce en toute lucidité. Mais, nous l'avons vu, si ce libre choix du mal correspond pour Kant au mal radical dans la nature humaine, il ne peut en être question chez Fichte. Celui-ci s'en tient à sa définition du mal radical (radikales Übel) comme pure inertie, ce qui justifie le reproche de philanthropisme que lui fait Schelling. Si dans les écrits de Fichte que nous analysons ici, on note quelques allusions à la perversité ${ }^{19}$, celui-ci s'empresse de dissiper le malentendu en en excluant résolument la possibilité pour le genre humain. Le mal vient toujours de la faiblesse, de l'impuissance à résister aux sollicitations de la nature sensible.

La perversion [Verkehrtheit] qui leur fait haïr le bien, parce que c'est le bien, et favoriser le mal par pur amour du mal comme tel, cette perversion qui seule pourrait exciter ma colère, je ne l'attribue à personne ayant figure humaine, car je sais qu'elle ne réside pas

\footnotetext{
${ }^{15}$ Fichte, System der Sittenlehre (1798), SW IV, p. 182, 198; tr., p. 175, 190.

${ }^{16}$ Fichte, System der Sittenlehre (1798), SW IV, p. 199, 202; tr., 191, 193.

${ }^{17}$ Immanuel Kant, Kritik der praktischen Vernunft, AK V, p. 59-60.

${ }^{18}$ Fichte, Die Bestimmung des Menschen (1800), SW II, 276.

${ }^{19}$ Fichte, Die Bestimmung des Menschen (1800), SW II, p. 270; System der Sittenlehre (1798), SW IV, p. 183.
} 
dans la nature humaine. Je le sais, pour tous ceux qui agissent de la sorte, il n'existe, au moment où ils agissent ainsi, ni bien, ni mal, mais uniquement de l'agréable ou du désagréable; ils n'agissent pas, en somme, de leur propre autorité, mais sous l'empire de la nature ${ }^{20} \ldots$

On le voit, entre le diabolique et la faiblesse humaine, il n'y a pas de moyen terme. Le mal, chez l'homme, ne vient que de l'ignorance délibérée de ce qu'est le véritable bien, à la réalisation duquel, lorsqu'on y est directement confronté, on ne peut se soustraire.

Le différend entre Fichte et Kant fait songer à celui qui oppose Platon et Saint-Augustin. Fichte refuse la corruption radicale de la nature humaine, au nom d'un humanisme qui adopte une vision moderne, voire cartésienne des choses. Il ne faut pas se surprendre, dans ces conditions, de le voir identifier le processus graduel de la prise de conscience du devoir moral au passage de l'« imagination » à l' « entendement». En effet, un examen serré du $§ 16$ de la Sittenlehre révèle de façon stupéfiante la manière toute cartésienne, sinon leibnizienne, dont Fichte décrit l'accession à la conscience de la loi: au départ cette conscience est « obscure » (dunkel, verdunkelt) et « indéterminée » (unbestimmt). Et la prise en charge par le Moi de sa propre liberté le conduit à une conscience « claire » (klar, $)$ 《 distincte » (deutlich) et « déterminée $^{21} \gg$ (bestimmt). Comme chez Leibniz, une connaissance claire et distincte du bien emporte immanquablement l'adhésion, sans dérogation possible. Il est vrai que ce n'est pas le seul trait que partage Fichte avec l'optimisme de Leibniz, tant s'en faut ${ }^{22}$.

Tout cela nous conduit à ma troisième thèse, qui a trait au modèle politique sous-jacent à la conception fichtéenne de la liberté dans son rapport au mal. Si l'explication du mal chez Fichte est largement tributaire de sa théorie de la liberté humaine, il importe d'examiner les tenants et aboutissants de cette théorie. À mes yeux, c'est dans l'Appendice au $§ 16$ de la Sittenlehre que le paradigme politique de la conception fichtéenne ressort avec le plus de clarté, en particulier lorsqu'il est question des trois « vices fondamentaux de l'homme ${ }^{23} »$ : paresse,

\footnotetext{
${ }^{20}$ Fichte, Die Bestimmung des Menschen (1800), SW II, p. 313-314; tr. (modifiée) M. Molitor, La destination de l'homme, Paris, Union Générale d'Éditions, 1965, p. 297. Voir également p. 298: « Il est vrai que la faute de ces hommes et leur indignité consistent justement en ce qu'ils sont ce qu'ils sont et qu'au lieu d'être libres et d'avoir de la personnalité ils se laissent aller à l'entraînement de l'aveugle nature.»

${ }^{21}$ Fichte, System der Sittenlehre (1798), SW IV, p. 178, 191-195; tr., p. 171, 183-187.

${ }^{22}$ Jean-Christophe Goddard, «Introduction » à Fichte, Essai d'une critique de toute révélation, Paris, Vrin, 1988, p. 25, 28. Voir également Jean Grondin, «Leibniz and Fichte», dans D. Breazeale et T. Rockmore, Fichte: Historical Contexts, Contemporary Controversies, Atlantic Highlands, Humanities Press, 1994, p. 181-190.

${ }^{23}$ Fichte, System der Sittenlehre (1798), SW IV, p. 202-203; tr., p. 193-194.
} 
lâcheté et fausseté (Faul-[heit], Feigheit, Falschheit). Laissons de côté la dernière, qui découle en fait de la lâcheté, pour nous concentrer sur les deux premiers vices. La paresse est le vice qui correspond directement à l'inertie, laquelle constitue pour Fichte l'essence même du mal (Übel) radical. Il s'agit par excellence de l'attitude de l'individu qui, refusant d'assumer sa liberté, s'enfonce dans les ornières de l'habitude. Quant à la lâcheté, elle est également reliée à l'inertie, mais cette fois-ci dans les rapports qu'entretient l'individu avec autrui. « La lâcheté est l'inertie qui, dans le rapport d'action réciproque avec d'autres, nous empêche d'affirmer notre liberté et notre autonomie ${ }^{24}$. \ C'est dire qu'autrui, dans la mesure où il cherche aussi à affirmer son autonomie, constitue de fait un obstacle à l'affirmation de la mienne.

Ce qu'il y a de singulier dans cette courte description qui est faite de ces deux vices dans l'Appendice, c'est qu'on retrouve dans chaque cas une allusion à Kant. Ce recours irait tout bonnement de soi si ce n'était que ces allusions renvoient en fait non pas aux textes classiques de Kant sur la philosophie morale, mais à deux opuscules de 1784, étroitement reliés à la politique. En effet, l'allusion à la paresse constitutive de la nature humaine fait implicitement référence à Kant. À considérer les choses de plus près, on se rend compte qu'elle se rattache à la Proposition IV de l«'Idée d'une histoire universelle au point de vue cosmopolitique » où il est question de cette fameuse « insociable sociabilité ». Dans ce passage, Kant affirme en effet que la nature prend les moyens requis pour sortir l'homme de son état de paresse initiale et le faire accéder à l'Aufklärung ${ }^{25}$. La seconde allusion à Kant se trouve dans l'explicitation du second vice, la lâcheté, et se rattache, elle aussi, au thème des Lumières. Encore que l'allusion ne soit qu'implicite, la phrase suivante nous fait immédiatement songer au texte de Kant «Qu'est-ce que les Lumières? »: « Je recule devant l'effort de penser par moi-même ${ }^{26}$. » Ce motif se place en effet sous le signe de la lâcheté parce que la renonciation à penser par soi-même ressortit à la thématique de l' « action réciproque avec d'autres ». Renoncer à penser par nous-mêmes est d'autant plus invitant que d'autres s'empressent de penser à notre place et de nous imposer leur autorité.

Avant d'aborder la signification précise de ces deux emprunts à Kant, qui ont à première vue quelque chose de banal, il y a d'abord lieu de s'interroger sur leur provenance. C'est que

\footnotetext{
${ }^{24}$ Fichte, System der Sittenlehre (1798), SW IV, p. 202; tr., p. 193.

${ }^{25}$ Immanuel Kant, « Idee zu einer allgemeinen Geschichte in weltbürgerlicher Absicht », AK VIII, p. 21; tr. L. Ferry, «Idée d'une histoire universelle au point de vue cosmopolitique », dans Kant Oeuvres philosophiques II, Paris, Gallimard, Biblioth. de la Pléiade, 1985, p. 192. Voir Fichte, Einige Vorlesungen über die Bestimmung des Menschen (1794), SW VI, p. 343; tr. J.-L. Vieillard-Baron, La destination du savant, Paris, Vrin, 1994, p. 88.

${ }^{26}$ Fichte, System der Sittenlehre (1798), SW IV, p. 202; tr. (modifiée), p. 194.
} 
l'Appendice de Fichte est explicitement consacré à la «thèse kantienne d'un mal radical dans $1^{\prime}$ homme ${ }^{27} \gg$. Si bien que l'on s'attendrait à ce que Fichte s'inspire au premier chef du texte de la Religion de même que de la façon dont le thème de la liberté transcendantale est développé dans la première Critique. Après tout, pour Kant le mal radical est affaire de libre arbitre, de liberté individuelle, voire de conscience intime. En d'autres mots, si le mal réside dans le renversement de l'ordre des maximes, le problème doit d'abord et avant tout être traité d'un point de vue gesinnungsethisch, du point de vue de la conviction profonde, sans égard aux considérations relatives à la réalisation effective du bien et du mal. Or, les opuscules de 1784 se placent sur le plan phénoménal, au niveau de l'expérience empirique. Et si la « liberté » y est invoquée, elle n'est pas interrogée comme problème métaphysique, mais seulement dans l'optique de ses conditions concrètes de réalisation. Le changement de registre est ici considérable et il nous appartient dès lors de retracer l'aspect sous lequel le thème du mal radical (l'inertie) est susceptible d'apparaître lorsqu'il est abordé à travers des concepts empruntés à la politique.

Nous avons noté ci-dessus que la liberté est envisagée chez Fichte d'un point de vue dynamique, au sens où la volonté s'inscrit d'entrée de jeu dans un rapport de forces au sein duquel elle est tenue de s'affirmer. Tout comme dans le texte de Kant sur les Lumières, la liberté est d'abord affaire de résolution et de courage: sapere aude! selon la devise des Lumières. Mais il y a plus: dans l'opuscule en question, la liberté est à ce point limitée par son environnement que sa réalisation ne tient pas uniquement au fait qu'on se décide à l'assumer, mais exige aussi qu'un espace de jeu lui soit consenti. Pour le dire autrement, la liberté doit à ce niveau être concédée à l'individu par l'autorité politique. On sait que le texte de Kant est un plaidoyer adressé au prince, en l'occurrence à Frédéric le Grand, afin qu'il écarte ou maintienne à l'écart les obstacles (Hindernisse) et les limitations (Einschränkungen) à la liberté du citoyen. À plusieurs reprises, Kant y souligne que la réalisation de l'autonomie politique dépend de la part de l'autorité politique d'un « laisser libre » (Freiheit lassen, frei lassen). Sans la levée de ces obstacles imposés tout naturellement par l'instance politique, les Lumières ont peu de chances de se propager. À l'évidence, Kant ne plaide pas pour l'élimination de toute contrainte, mais seulement pour l'abrogation des mesures qui, comme la censure, étouffent l'espace public, et ce afin que l'individu puisse avoir le libre usage de son entendement. Au sein du rapport dynamique dans lequel vient prendre place l'Aufklärung du " public », la liberté doit donc être considérée sous deux angles: elle doit être prise en main par l'individu, d'où l'injonction sapere aude! mais aussi pouvoir se déployer dans un contexte non répressif. Du moins, c'est ainsi que l'entend Kant: « Les hommes se dégagent eux-mêmes peu à peu de leur grossièreté, si seulement

${ }^{27}$ Fichte, System der Sittenlehre (1798), SW IV, p. 198; tr., p. 190. 
on ne s'évertue pas à les y maintenir ${ }^{28}$. » Si on transpose cette situation dans les termes du $§ 16$ de la Sittenlehre, on dira que le Moi doit s'élever à l'autonomie par ses propres forces, pour autant que le Non-Moi, qui ne relève aucunement de la liberté du Moi, n'oppose pas d'obstacle insurmontable à cette émancipation.

Le parallèle établi ici entre les textes de Fichte et de Kant peut paraître audacieux, pour ne pas dire arbitraire. Mais à regarder les choses de plus près, il n'est pas interdit de penser que Fichte s'est inspiré de l'opuscule de Kant. En effet, immédiatement après l'énoncée du mot d'ordre des Lumières, Kant enchaîne en faisant état précisément --et dans les mêmes termes-- des deux vices qui conditionnent à la fois la structure binaire de son argumentation et les deux aspects (individuel/communautaire) que revêt le mal moral chez Fichte:

La paresse [Faulheit] et la lâcheté [Feigheit] sont les causes qui expliquent qu'un si grand nombre d'hommes, alors que la nature les a affranchis depuis longtemps de toute direction étrangère (naturaliter maiorennes), restent cependant volontiers, leur vie durant, mineurs; et qu'il soit si facile à d'autres de se poser comme leurs tuteurs ${ }^{29}$.

Le plus frappant dans tout cela, c'est que dans son exposé sur la lâcheté, Fichte ne manque pas non plus de souligner qu'un tel vice ne peut avoir libre cours que grâce à la complicité d'un prince tyrannique. Nous aurons d'ailleurs à revenir plus loin sur la question de l'abus de pouvoir en politique. Qu'il suffise d'ajouter pour clore la discussion sur l'inspiration politique de Fichte dans sa théorie de la liberté que cette dernière est conçue selon le schème de réalisation progressive propre aux Lumières. On pourrait bien sûr être tenté de rétorquer que les Lumières concernent toutes les variantes de l'obscurantisme, c'est-à-dire tout autant les question de dogmatisme religieux que de tyrannie politique. Mais le fait est que Kant s'adresse d'abord et avant tout à l'autorité politique, seule en mesure de mettre un terme à toute forme d'autorité dogmatique, et au premier chef à celle de la religion. À ses yeux, l'Aufklärung interpelle toujours d'abord l'État ${ }^{30}$.

\footnotetext{
${ }^{28}$ Kant, « Was ist Aufklärung? », AK VIII, p. 41; tr., p. 216.

${ }^{29}$ Kant, « Was ist Aufklärung? », AK VIII, p. 35; tr., p. 209.

${ }^{30}$ Immanuel Kant, Der Streit der Fakultäten, AK VII, p. 89; tr. A. Renaut, Le conflit des facultés, dans Kant, Oeuvres philosophiques III, p. 900. Jean-Christophe Goddard a justement remarqué que la conception fichtéenne de la liberté repose sur une « authentique philosophie de l'effort », dont l'inspiration est sans doute à chercher dans le fait que Fichte « partageait l'éthos religieux des révolutionnaires français, voyant le divin dans l'action même des hommes... ». Voir son «Introduction » à l'Essai d'une critique de toute révélation, p. 25-26.
} 
Nous avons trouvé les sources kantiennes de Fichte en regard de la théorie du mal radical là où nous nous y attendions peut-être le moins: dans les opuscules de 1784. À vrai dire, Fichte s'y réfère parce qu'ils rendent compte de sa façon de voir les choses, si bien qu'il n'attendra pas de prendre connaissance de l'essai de Kant sur le mal radical (1792) pour établir son point de vue de manière définitive. Bien au contraire, au moment de la parution de cet essai, l'essentiel de sa position est déjà arrêté, comme on peut s'en rendre compte par exemple en consultant le sermon Sur l'amour de la vérité de 1791-1792, ou encore la première édition de l'Essai d'une critique de toute révélation $^{31}$. Certes, le mal est imputable à l'homme libre, mais ce dernier ne peut être envisagé comme sciemment et délibérément mauvais. Il commet le mal simplement parce qu'il laisse libre cours en lui à la nature, à la sensibilité. Là s'arrête aussi sa responsabilité. De cette manière, il est difficile d'admettre avec Fichte que le $\$ 16$ puisse constituer un approfondissement de la théorie kantienne du mal moral. J'ai donc proposé une lecture du texte à rebrousse-poil afin de cerner les motifs qui semblent avoir échappé à Soller et Ivaldo, et qui nous montrent un Fichte renouant, par-delà Kant, avec la tradition humaniste. Schelling d'ailleurs ne s'y est pas trompé, lui qui en 1806 dans sa mise au point sur la « doctrine fichtéenne » n'a pas manqué de remarquer que celle-ci se fusionne avec l'Aufklärung ambiante ${ }^{32}$. La question est alors de savoir si Fichte demeurera fidèle à cette vision humaniste des choses jusqu'à la fin de sa carrière. Reinhard Lauth, pour sa part, en doute et croit déceler en l'année 1806 une révision en profondeur du point de vue de Fichte sur le mal radical. Ce qui nous conduit à la seconde étape de notre recherche.

\section{II - Examen de la thèse de Reinhard Lauth}

Ce qu'il y a de remarquable dans la thèse de Lauth, c'est qu'il prétend que Fichte aurait, vers la fin de sa carrière, modifié sa conception du mal radical pour la rapprocher de celle de Kant. Il admet donc implicitement que la théorie fichtéenne initiale, fondée sur l'inertie, ne représentait pas le point de vue de Kant, auquel Fichte sera plus tard amené à se rendre, par la force des choses. Mais, comme Lauth date la réorientation en question de l'année1806, cela

\footnotetext{
${ }^{31}$ Fichte, Zwei Predigten aus dem Jahre 1791, SW VIII, p. 266; tr. J.-C. Goddard, dans Fichte, La querelle de l'athéisme, Paris, Vrin, 1993, p. 260: «Se tenir pour corrompu, et se résoudre à le rester, est contre-nature. » De même, Fichte, Versuch einer Kritik aller Offenbarung, première édition (1792), SW V, p. 89 (passage inchangé en 1793); tr. J.-C. Goddard, Essai d'une critique de toute révélation, p. 115: « La plus grande déchéance des êtres raisonnables eu égard à leur moralité advient enfin lorsqu'il n'y a pas même la volonté de reconnaître une loi morale et de lui obéir, lorsque les tendances sensibles sont les seuls principes de détermination de leur faculté de désirer. »

${ }^{32}$ F. W. J. Schelling, Darlegung des wahren Verhältnisses der Naturphilosophie zu der verbesserten Fichteschen Lehre (1806), édition Cotta, I/7, p. 36.
} 
signifie que Fichte aurait paré d'avance au reproche adressé par Schelling dans son traité de 1809, selon lequel Fichte est incapable d'attribuer le mal radical à un « acte de la liberté » au plein sens du terme. Lauth décèle en fait chez Fichte une évolution semblable à celle qu'aurait connue la théorie kantienne du mal: de même que l'essai de 1792 sur le mal radical rompt avec la conception antérieure de Kant qui situe l'origine du mal dans le sensible, de même Fichte en serait venu pour sa part à envisager la mal radical non plus comme inertie mais comme émanant du libre arbitre qui s'affirmerait ouvertement contre le bien moral ${ }^{33}$.

Aux dires de Lauth, cette surprenante volte-face ne serait d'ailleurs pas liée à une évolution interne de la Wissenschaftslehre, mais bien plutôt à des circonstances extérieures, d'ordre politico-historique: Fichte aurait changé d'avis à propos de la présence du mal dans le monde à la suite de la chute du Saint Empire Germanique sous la poussée des troupes napoléoniennes. Aussi la réévaluation du problème du mal radical aurait-elle laissé des traces, non seulement dans les Discours à la nation allemande (1808), mais aussi dans la Théorie de l'État de 1813. Telles sont du moins les sources privilégiées de Lauth. Mais voyons tout d'abord comment est décrite cette figure du « mal extrême » qui s'introduit dans le monde sous la forme du despotisme absolu. L'extrait suivant présente l'avantage de marquer le passage entre le texte que nous avons privilégié jusqu'ici, le Système de l'éthique, et les écrits plus tardifs.

Dans le Système de l'éthique de 1798 il y a la conception d'une position absolue de l'arbitraire; et nous sommes en possession d'une Nachschrift du cours de Morale de 1796 où on trouve le même passage. Donc, c'est une conception qui date d'avant Napoléon -bien sûr, il y avait déjà Bonaparte comme général, il n’y avait pas encore Napoléon! Il y a une différence remarquable entre cette conception de l'arbitraire et celle que Fichte aura à la fin de sa vie, dans la Staatslehre de 1813. Il voit très bien la possibilité de faire un absolu du propre vouloir arbitraire, mais avant 1806, cela ne peut entrer dans la fonction d'une attitude volontairement opposée à la raison. C'est le fait nouveau à retenir après la chute du Saint Empire ${ }^{34}$.

Cette thèse de Lauth est du plus haut intérêt pour notre propos puisqu'elle souligne la présence chez le dernier Fichte de cet élément qui était absent jusque-là, à savoir l'acte libre de déroger

\footnotetext{
${ }^{33}$ Reinhard Lauth, « L'action historique d'après la philosophie transcendantale de Fichte » (1976), dans le même, Vernünftige Durchdringung der Wirklichkeit, Neuried, ars una, 1994, p. 385.

${ }^{34}$ Reinhard Lauth, en réponse à la question de $\mathrm{P}$. $\mathrm{Ph}$. Druet à la suite de la présentation de sa conférence " L'action historique... », in ibid., p. 404. On pourrait aussi citer le passage suivant, tiré de la conférence, p. 386: «Fichte découvre alors pleinement qu'au-delà de l'inertie paresseuse il y a aussi une autre possibilité accrue de contreraison. La volonté libre n'est pas déterminée par sa nature à réaliser la raison, elle peut aussi nier le but, afin de se poser absolument elle-même. Le libre-arbitre absolu peut se constituer luimême en fin suprême. »
} 
sciemment au commandement de la loi morale. Il serait donc instructif d'analyser le corpus fichtéen mis à contribution ici afin d'évaluer la portée de cette percée théorique de même que son bien-fondé, du moins en ce qui a trait à son articulation précise dans les textes mentionnés cidessus.

Lorsque l'on s'intéresse à la portée de sa nouvelle approche du problème du mal radical, il faut bien remarquer que cette conception inédite chez Fichte porte la marque du contexte de sa genèse: cette figure du mal radical ne serait pas le fait de l'humanité entière, mais uniquement de certains êtres d'exception. En fait, Fichte s'insurge ainsi contre les prétentions de Napoléon au moment où celui-ci devient Premier Consul. C'est la prétention illimitée de cette affirmation de soi qui soulève son indignation. Napoléon veut tout simplement s'ériger en maître du monde et il incarne un paroxysme d'immoralité (le mal radical) en cherchant à maintenir tous les êtres humains autour de lui dans la servitude. Le mal chez ce genre de tyran ne tient donc pas à la simple inertie (paresse, lâcheté et fausseté) du commun des mortels, mais au contraire à l'autoaffirmation absolue de son propre libre arbitre envers et contre tous, dans le but d'empêcher les hommes de s'éveiller à leur liberté.

Le problème, pourtant, avec cette nouvelle approche, c'est qu'elle restreint singulièrement le champ du mal radical, de sorte que la portée philosophique d'une telle détermination s'en trouve réduite. La théorie kantienne généralisait en effet l'inclination au mal radical à l'ensemble du genre humain: il y est nommément question du mal radical « dans la nature humaine ». Et la première théorie de Fichte ne prétendait pas accéder à un moindre niveau de généralité: l'inertie rattachée à la nature sensible constitue la condition de tout être humain, voire de tout être raisonnable fini. Mais, parce qu'elle s'adresse à des êtres hors du commun dont la volonté est particulièrement forte, la conception amendée du dernier Fichte ne touche qu'une classe d'hommes très particulière. En revanche, pour ce qui est de la condition de l'humanité prise dans son ensemble, sa position ne semble pas évoluer da façon marquante. Il conserve sa confiance dans un genre humain dont la destination profonde serait d'emblée tournée vers le bien. Si, par exemple, l'Anweisung (texte précédant le tournant) nous interdit de considérer que l'homme est au départ un pauvre pécheur, mais le présente plutôt comme une être bon, le dixième Discours à la nation allemande de 1808 ne dit pas autre chose: « [C]'est une grossière insulte à la nature humaine que de prétendre que l'homme est né pécheur ${ }^{35}$. » À ce niveau de généralité, on ne dénote donc aucune rupture significative avec les textes que nous avons étudiés.

\footnotetext{
${ }^{35}$ Fichte, Reden an die deutsche Nation (1808), SW VII, p. 421. Et Anweisung zum seligen Leben, SW V, p.565. D'ailleurs, tout comme dans les textes antérieurs, la méchanceté est ici écartée au profit de l'« incompréhension ». Voir Reden.., p. 476: «Que l'on songe moins à la méchanceté réfléchie et à la trahison. L'incompréhension et l'inertie suffisent dans pratiquement tous les cas à expliquer les événements. »
} 
L'homme est destiné au bien et il ne tient qu'à lui de le réaliser.

Quant à l'inscription de la nouvelle conception du mal radical dans la Staatslehre de 1813, il y aurait lieu de s'interroger d'abord sur la nouveauté du thème de la volonté égoïste qui s'y fait jour. À ce sujet, Lauth indique à juste titre que le thème de l'auto-affirmation formelle et vide du libre arbitre n'est pas nouveau. Tout dépend dès lors de l'ampleur de la réévaluation de ce thème. En effet, Lauth a bien repéré dans le $\S 16$ de la Sittenlehre le problème de l'affirmation absolue de soi (Selbstständigkeit). La figure du tyran y est même évoquée, comme l'a laissé entrevoir ci-dessus notre examen des trois vices fondamentaux de l'homme au $§ 16$. Dans l'explication de la lâcheté et de la fausseté, il est question du despote qui fournit un prétexte à la créature inerte pour demeurer dans son état d'esclavage, et utilise les hommes comme moyens au profit de l'augmentation de sa puissance --ce à quoi, du reste, ils se plient le plus souvent de bon gré, ajoute Fichte.

Mais alors, il faut s'interroger sur la nature du saut qualitatif qui sépare la Sittenlehre de 1798 de la Staatslehre de 1813. Certes c'est un plus grand mal de faire obstacle à ce que les hommes accèdent à la liberté, que de simplement se complaire dans sa propre impuissance. Mais le fait est que Fichte a clairement envisagé cette possibilité au $§ 16$ sans pourtant y accorder un statut spécial, sans y voir à tout le moins la quintessence du mal radical. Après tout, l'esclave est complice de son maître, avons-nous déjà noté. Est-ce à dire que l'entrée en scène de Napoléon et la défaite de l'Allemagne auraient représenté aux yeux de Fichte un fait à ce point exceptionnel dans l'histoire de l'humanité qu'il aurait commandé un rajustement de la théorie? Ce serait dire que Napoléon incarne le paroxysme de tout ce qui a valu jusque-là comme mal radical.

Voyons à l'aide de la Staatslehre de 1813 le portrait qui nous est dressé de cet empereur que Fichte d'ailleurs refuse de nommer par son nom. Il est vrai qu'il est peu flatteur, et ceci non seulement parce que Napoléon a réduit le peuple allemand à l'état de servitude, mais aussi --et peut-être d'abord-- parce que Napoléon est pour la France elle-même un usurpateur. Ce détail n'est pas sans importance pour Fichte, puisque les origines corses de l'empereur nous rappellent qu'il est issu d'un peuple « sauvage » et sans éducation. Bonaparte n'a pas dans sa jeunesse développé un sentiment d'appartenance à l'égard du peuple français, qu'il lui était dès lors loisible de considérer comme un ensemble informe de forces auquel il convient de donner une direction. S'il a pu se considérer comme l'homme de la situation, c'est qu'il s'agit d'un être exceptionnel, à deux points de vue: il se distingue en effet, selon Fichte, du commun des mortels d'abord par sa clairvoyance politique, et ensuite par le caractère inébranlable de sa volonté, prête à tous les sacrifices pour pouvoir s'affirmer de manière absolue. La figure ainsi dépeinte est d'une certaine manière monstrueuse car, ayant accédé à une telle position de force, la préoccupation première du prince aurait dû consister non pas à se soumettre tous les peuples de 
la terre, mais à instituer dans son propre pays une « éducation nationale » du genre de celle que décrivent les Discours. Or Napoléon, estime Fichte, s'en tient à la tyrannie pure et simple.

Nous sommes maintenant parvenus à l'étape de notre examen de la Staatslehre qui nous permet de vérifier le bien-fondé de la thèse de Lauth. Fichte est très précis sur les raisons qui expliquent la voie qu'a empruntée Napoléon. Celui-ci, n'ayant pas joui dans son enfance d'une éducation digne de ce nom -- pas même à la française! -- est en fait incapable de s'élever au point de vue de la moralité pure. Il en reste au contraire au plan de l'auto-affirmation absolue, sans autre but que d'assurer et d'augmenter sa propre puissance. Le texte est à cet égard on ne peut plus explicite. Il n'y a pas chez Napoléon la moindre « étincelle d'une conviction authentique », il n'a pas la moindre idée de la « destination morale supérieure de l'homme ${ }^{36}$ ». Autant dire qu'il lui est d'emblée interdit de considérer même le projet d'une éducation nationale, ne s'étant jamais élevé à la conscience claire du devoir moral. Si bien que la description que nous en donne Fichte ne se distingue en rien du portrait du tyran esquissé au $§ 16$. Le mal radical, tout comme son acception de base fondée sur l'inertie, relève encore et toujours de l'ignorance, et non d'une libre décision pour ce qui est immoral. Napoléon n'ayant aucune idée de la moralité, le référence de Lauth à la Staatslehre ne soutient pas véritablement sa thèse d'une réévaluation du statut et de la nature du mal radical dans le sens de Kant. Par l'étendue de sa puissance, l'empereur est sans conteste pour l'époque l'homme qui commet le plus grand mal, mais sans en avoir véritablement conscience. De ce point de vue, la conception fichtéenne n'a pas changé.

Peut-être Lauth songe-t-il à d'autres textes du dernier Fichte à l'appui de la thèse selon laquelle l'auteur des Discours en serait venu à admettre que le libre arbitre puisse se déterminer au mal en toute connaissance de cause. Mais revenons à Kant pour examiner la terminologie dont il se sert pour désigner l'attitude de celui qui prend délibérément le contrepied de la loi morale en donnant préséance à l'amour de soi. Il s'agit, on s'en souvient, du concept de « perversion » qui, loin de mener selon Kant au diabolique, désigne simplement l'inversion de l'ordre des maximes. Or, dans les Cinq leçons sur la destination du savant de 1811, Fichte reprend à son compte le concept de perversion, ce qui pourrait en apparence appuyer le thèse de Lauth, avec cette nuance cependant qu'il l'envisage dans un sens qui diffère, sur un point crucial, de l'acception kantienne. Voici un passage hautement significatif de la première Leçon.

...tout ceci qui nous apparaît comme un malheur [Unheil] n'est en fait aucunement présent, seul le salut [Heil] et le progrès du salut est réellement présent dans le monde

\footnotetext{
${ }^{36}$ Fichte, Die Staatslehre, oder über das Verhältnis des Urstaates zum Vernunftreiche, in Vorlesungen (1813), SW IV, p. 424, 430; cf. p. 425.
} 
éternel. Perverse et corrompue, l'humanité l'est depuis le tout début et elle va le demeurer encore longtemps; du reste cette corruption n'était d'entrée de jeu rien d'autre que la mé-compréhension, l'opposition -- et sa manifestation -- à l'égard du vrai qui est toujours présent dans le monde ${ }^{37}$.

Si le mal dans le monde n'est qu'une apparence, voire un manque, il va de soi que la corruption et la perversion ne peuvent s'appuyer sur quelque instance positive que ce soit, comme par exemple sur une décision éclairée. Le texte est limpide à ce sujet. Bien sûr, le mal constitue un obstacle, une opposition au bien et au vrai, mais Fichte prend soin d'indiquer que cette opposition se fonde en premier lieu sur un malentendu (Missverständnis). L'absence d'une saisie claire et distincte du bien est à nouveau ici à l'origine du mal. Tout comme dans la Sittenlehre de 1812, Fichte développe ici un concept de résistance au bien: l'inertie a beau se manifester sous la forme de la paresse, il n'en demeure pas moins qu'elle représente pour le progrès vers le bien une entrave, une force de résistance ${ }^{38}$. Mais ce qu'il faut surtout retenir de l'extrait cité -- de même que de l'ensemble du passage en question -- c'est que cette inertie est entretenue par un malentendu sur la destination véritable de l'homme. On le voit, c'est encore ici l'absence ou le dévoiement de la dimension cognitive qui est à l'origine du mal moral. Fichte n'abandonnera jamais cette composante intellectuelle, à la décharge de l'être humain fini. Celui-ci n'est pas foncièrement méchant et ne peut en toute lucidité se considérer comme radicalement mauvais (radical schlecht ${ }^{39}$ ).

Nous avons amorcé cette étude en signalant le trait que Fichte partage avec la conception kantienne du mal radical: la présence d'une mauvaise maxime ne peut s'expliquer qu'en rapport avec la liberté humaine. En fin de parcours, il y a lieu de revenir sur la nature de ce rapport entre le mal et la liberté qu'on retrouve tant chez Kant que chez Fichte et --bien que nous ayons été dans l'impossibilité de le faire intervenir dans le développement de l'argument-- chez Schelling. Chez Kant, la préoccupation première de la théorie du mal moral réside dans la nécessité de sauvegarder l'imputabilité, et ce à tout prix. Le choix du mal doit donc être un acte libre, effectué en toute connaissance de la loi morale. Celle-ci est d'ailleurs un « fait» de la raison, donné d'emblée à tout un chacun en sorte que personne ne puisse s'en prétendre privé. Kant qualifie aussi cette conscience de la loi morale d'Einsicht, présente à la conscience de tout être raisonnable. Du point de vue de la théorie morale, ce fait constitue, comme on sait, la ratio

\footnotetext{
${ }^{37}$ Fichte, Fünf Vorlesungen über die Bestimmung des Gelehrten (1811), SW XI, p. 159.

${ }^{38}$ Fichte, Das System der Sittenlehre (1812), SW XI, p. 61.

${ }^{39}$ Fichte, Die Anweisung zum seligen Leben, SW V, p. 564.
} 
cognoscendi de la liberté, laquelle du point de vue de la philosophie transcendantale demeure un concept purement problématique. Fichte, pour sa part, ne renonce pas plus que Kant à la dimension de responsabilité constitutive d'une juste saisie du mal moral. Mais il se dote d'instruments théoriques beaucoup plus puissants que ceux que Kant avait à sa disposition --un timide recours à l'intelligible. Comme l'a bien remarqué Marco Ivaldo, la théorie fichtéenne du mal radical repose sur une philosophie transcendantale pleinement constituée. Celle-ci est aussi en mesure de fournir une explication en bonne et due forme de la présence du mal dans le monde: il s'agit de l'inertie comme condition initiale de tout être humain. Par-delà Fichte, Schelling salue la radicalité du geste de Kant qui rattache le choix du mal directement à un acte de la liberté. Car pour Schelling également la responsabilité morale est importante, avec cette différence qu'il l'insère dans un contexte théorique qui n'est manifestement plus celui de la philosophie transcendantale, mais plutôt celui d'une grandiose construction métaphysique à fortes connotations religieuses. Certes, son cadre explicatif est plus riche, mais il soulève également beaucoup de questions. En effet, Schelling entreprend de réconcilier la nécessité de la présence du mal dans le monde -- afin que Dieu puisse se révéler -- avec la culpabilité de l'homme pour le mal qu'il commet en ce monde. Aussi le célèbre traité de 1809 sur la liberté humaine demeure-t-il aux prises avec les apories pratiquement insolubles que provoquent ces deux exigences contradictoires.

Dans ce contexte, la théorie fichtéenne du mal, qui occupe une position mitoyenne entre celles de Kant et Schelling, offre l'avantage de procurer une schème d'explication plausible du mal, tout en respectant dans une certaine mesure la dimension d'imputabilité. On passe de la sorte du mystère que représentait chez Kant le choix lucide du mal, à l'énigme que pose pour Fichte un sujet fini qui s'élève librement à la lucidité. 